\title{
Privileged-Perspective Realism in the Quantum Multiverse
}

\author{
Forthcoming in The Foundation of Reality: Fundamentality, Space and Time. Marmodoro, A., G. \\ Darby. And D. Glick (eds.) Oxford University Press. \\ (please cite final version)
}

\section{Nora Berenstain}

\begin{abstract}
Privileged-perspective realism (PPR) is a version of metaphysical realism that takes certain irreducibly perspectival facts to be partly constitutive of reality. PPR asserts that there is a single metaphysically privileged standpoint from which these perspectival facts obtain. This chapter discusses several views that fall under the category of privileged-perspective realism. These include presentism, which is PPR about tensed facts, and non-multiverse interpretations of quantum mechanics, which the chapter argues, constitute PPR about world-indexed facts. Using the framework of the bird perspective and the frog perspective, it argues that PPR views methodologically treat the frog perspective as metaphysically primary. This chapter considers case studies of metaphysical interpretations of special relativity and quantum mechanics in order to demonstrate that such motivations for PPR are non-naturalistic. Further, it considers psychological factors that motivate the appeal of PPR views and offers naturalistic explanations of why we should not expect them to produce an adequate metaphysics of science.

Keywords: quantum mechanics, metaphysics of space-time, quantum multiverse, Everett interpretation, Copenhagen interpretation, presentism, special relativity, perspectival facts, metaphysics of science
\end{abstract}

\subsection{Metaphysics and Perspectival Facts}

“Consider a world made up of pointlike particles moving around in three-dimensional space. In four-dimensional spacetime - the bird perspective - these particle trajectories resemble a tangle of spaghetti. If the frog sees a particle moving with constant velocity, the bird sees a straight strand of uncooked spaghetti. If the frog sees a pair of orbiting particles, the bird sees two spaghetti strands intertwined like a double helix. To the frog, the world is described by Newton's laws of motion and gravitation. To the bird, it is described by the geometry of 
the pasta - a mathematical structure. The frog itself is merely a thick bundle of pasta, whose highly complex intertwining corresponds to a cluster of particles that store and process information." (Hut, Alford, and Tegmark 2006, p. 770)

"I am a Tralfamadorian, seeing all time as you might see a stretch of the Rocky Mountains. All time is all time. It does not change. It does not lend itself to warnings or explanations. It simply is. Take it moment by moment, and you will find that we are all, as I've said before, bugs in amber." (Kurt Vonnegut Jr. 1969)

Metaphors of the bird perspective and the frog perspective allow us to visualize certain aspects of the metaphysical pictures that result from our efforts to make sense of various physical systems, structures, and phenomena. Alternatively called the God's-eye view and the ant's-eye view (Silberstein, Stuckey, and McDevitt 2018), these two viewpoints delimit the perspective of an observer viewing or modeling a system from an external vantage point and an observer experiencing that system from within, respectively. When a physicist studies the equation for Newtown's second law of motion, they theorize the laws from the external, mathematical bird perspective. When they watch someone throw a Frisbee, they observe its motion from the internal frog perspective. As sentient observers, we are capable of making inferences and observations from either or both of these perspectives. We form our quotidian picture of the world by combining inferences and knowledge obtained from both. Often, there are tensions between these two pictures. When such tensions arise then, which picture ought to be ascribed metaphysical priority? 
In this chapter, I address two applications of the question of whether the bird view or the frog view is metaphysically fundamental. I raise several concerns about approaches to metaphysics of science that prioritize the fundamentality of the frog view over the bird view. Specifically, I discuss shortcomings of this approach with attention to two families of metaphysical views: (1) presentist interpretations of special relativity and (2) non-multiverse interpretations of quantum mechanics. I locate these approaches in the intellectual tradition of a priori metaphysics that aims to preserve 'common-sense' intuitions and argue that they are not compatible with a naturalistic methodology that is attentive to the history of modern science. I also suggest that the fundamentality they ascribe to the content of our intuitions and phenomenal experiences is in tension with an evolutionary understanding of the development of our cognitive faculties and what Silberstein, Stuckey and McDevitt (2018) have called our resulting dynamical bias.

Consider the distinction between the bird view and the frog view in terms of the metaphysics of perspectival facts. A perspectival fact is a fact that obtains from within the frog view. Here, the term "perspectival fact" is used to refer to any fact expressed by a proposition whose truth is relative to the perspective(s) or location(s) within the world of some (possible) observer(s). Firstpersonal facts (e.g., "I am a professor"), spaced facts (e.g., "The Air \& Space Museum is three miles away from here"), and tensed facts (e.g., "It is snowing in Cedar Rapids") are examples. Contrast these with non-perspectival facts, such as "The author of "Against PrivilegedPerspective Realism in the Quantum Multiverse' is a professor," "The Air \& Space Museum is three miles away from Dupont Circle," and "It is snowing in Cedar Rapids at 2:18 pm EST on January 28, 2018." We may then ask, are the perspectival natures of the first group of facts features of fundamental metaphysical reality or merely features of the statements by which they 
are described? As Fine $(2005$, p. 261) puts it, "Is reality itself somehow tensed, or spatiocentric, or first-personal, or is it merely that we describe a tenseless or spatially uncentered or impersonal reality from a tensed or spatiocentric or first-personal point of view?"1 The question is whether some statements of irreducibly perspectival facts express metaphysical truths or whether metaphysical truths are only non-perspectival.

Privileged-perspective realism (PPR) accepts that certain irreducibly perspectival facts are constitutive of reality and asserts that there is a single metaphysically privileged standpoint from which these perspectival facts obtain. It is possible to hold a PPR view about any sort of perspectival fact. If I accept PPR about first-personal facts, I believe that certain first-personal facts are constitutive of reality, and that there is only one first-personal standpoint (presumably, my own) from which such facts obtain. Thus, the only first-personal facts that are constitutive of reality are the ones concerning me. While this particular version of PPR may seem odd, some forms of PPR are widely defended. Presentism, for instance, is PPR about tensed facts. Many of its defenders find it to be self-evidently true given our experience of time. Presentists believe that reality itself is tensed and that the irreducibly tensed facts that are constitutive of reality are the ones that obtain in relation to the present. Of course, this view is not without problems.

\subsection{The Argument from Special Relativity against PPR}

\section{about Tensed Facts}

\footnotetext{
${ }^{1}$ For Fine, metaphysical truths are irreducible, so the question becomes whether there are perspectival facts that are not reducible to non-perspectival facts.
} 
One of the strongest challenges to PPR about tensed facts arises from the argument that it is incompatible with special relativity. Putnam (1967) presented an early version of this argument. Stein (1968) and Saunders (2002) have both offered critiques and defended reformulated versions of it. Fine (2005) explicates how mathematical and theoretical considerations create difficulties for the intuitively appealing metaphysical view of PPR about tensed facts.

I discuss Fine's introduction of four metaphysical principles that provide conceptual scaffolding for characterizing the differences between privileged-perspective realism and antirealism about perspectival facts. In his discussion of McTaggart's classic (1908) argument for the unreality of time, Fine demarcates the four conceptual principles that a view about time can accept: realism, absolutism, neutrality, and coherence. He defines each principle as follows $(2005,271)$ :

1. Realism-Reality is constituted (at least in part) by tensed facts.

2. Neutrality-No time is privileged, the tensed facts that constitute reality (if any do) are not oriented towards one time as opposed to another.

3. Absolutism-The composition of reality is an absolute matter, i.e. not relative to a time or other form of temporal standpoint.

4. Coherence-Reality is not contradictory, it is not constituted by facts with incompatible content.

While the four principles are together incompatible, the combination of any three results in a consistent metaphysical view. Different combinations of these principles produce the conceptually possible views of tensed facts. Anti-realist views about tensed facts such as eternalism, for example, maintain Absolutism, Coherence, and Neutrality. Eternalism gives up 
Realism about tensed facts, as it accepts that reality contains no tensed facts that cannot be adequately expressed by or reduced to tenseless facts. Thus, rather than "It is snowing in Cedar Rapids" uttered at 2:18 pm EST on January 28, 2018 expressing a fact that is part of metaphysical reality, "It is snowing in Cedar Rapids at 2:18 pm EST on January, 28, 2018" uttered anywhere would express such a fact. No feature of reality is left out by including the fact expressed by the latter statement in the set of metaphysical facts but not that expressed by the former. That all metaphysical facts expressing the temporal location of an event or the temporal distance between events hold independently of temporal location or spatio-temporal frame of reference for the eternalist reflects the view's Neutrality about tensed facts.

PPR views, on the other hand, such as pre-relativistic presentism, maintain Realism, Absolutism, and Coherence. The PPR view is realist because it takes reality to contain tensed facts that are not reducible to or expressible by any tenseless facts. It is absolutist because these facts hold absolutely rather than relative to a specific perspective. The view is coherent because it does not countenance inconsistent facts as part of reality. Lastly, the view gives up Neutrality because it privileges a specific perspective or stand point — namely, the present one — from which the irreducibly tensed facts that are constitutive of reality hold.

In his discussion of Realism about tensed facts, Fine (2005) distinguishes between what he calls ontic presentism and factive presentism. Ontic presentism is the ontological view that only present things exist, while factive presentism is the view that reality is partly constituted by irreducibly tensed facts. Ontic presentism thus presupposes factive presentism. When philosophers consider special relativity's compatibility with presentism, they tend to address the ontological question of what the presentist should take to be real. But Fine introduces the 
following as a more basic question that the presentist must answer: Since the presentist believes in tensed facts, what facts should the presentist take to be tensed in light of special relativity?

The obvious but pre-theoretic answer would be just those facts that obtain with respect to the present moment. But special relativity’s preclusion of an absolute notion of simultaneity poses a problem for the pre-relativistic conception of a tensed fact as one that obtains relative to a time. Consider the following: If $e$ and $f$ are events, then the propositions that they are occurring now are tensed. If it makes sense to say that the proposition that $e$ is occurring now and the proposition that $f$ is occurring now are true at any given time, then we can ask if they are true at the same time. For the propositions to be true at the same time is for the events to be simultaneous. The pre-relativistic concept of tense presupposes absolute simultaneity, but since there is no absolute simultaneity under special relativity the tense-theoretic realist must replace the temporal moment as that with respect to which a tensed proposition obtains.

There are two options for what the realist about tensed facts may take to be that with respect to which a tensed proposition holds: a space-time point, or an inertial frame of reference plus a time. If we evaluate a tensed proposition with respect to a space-time point, then a tensed proposition would be one that declares that a given event holds (or is earlier or later than) herenow (Stein 1991). Harrington (2008) defends a version of this view. Alternatively, since each frame of reference gives rise to a framework of times, a tensed proposition could be evaluated with respect to a frame plus one of its times. Tensed propositions would then be those that say a given event is now or that a given thing is now-at-rest, relative to the frame of reference. Fine concludes that neither of these two options is feasible.

The first option, what Fine calls the locational account, faces a significant problem. Because it involves the merging of temporal and spatial relativity, it fails to preserve one of the primary 
motivations for presentism, namely the sense that there is an important metaphysical distinction between time and space. The presentist accepts that there is a metaphysically privileged point (or, more precisely, hyperplane) of time but no corresponding metaphysically privileged area of space. Tensed facts are partially constitutive of reality, while facts that are true relative to a spatial location - spatiocentric facts - are not. Thus, if the presentist accepts the collapse of temporal and spatial relativity into spatio-temporal relativity, they must relinquish one of the primary motivations for their view.

Aside from the locational account, the other option for the presentist is to evaluate tensed facts with respect to an inertial frame of reference plus a time. But both accounts face the problem of arbitrariness. If only one inertial frame + time is to be constitutive of reality, which one is it? Fine suggests that the only plausible answer seems to be "the one that I occupy." But there can be no good reason to prefer my inertial frame to any other as the metaphysically privileged perspective with respect to which tensed facts hold.

If I accept the locational account, then the metaphysically privileged location from which to answer a tensed question will be the space-time point from which I ask it. If you ask the question from a space-time point that is within my light-cone (i.e._-in my absolute past or future), I may have a presentist reason to exclude your frame of reference from reality, since you pose the question from a different time than my present. But suppose you instead ask the question from a point that is only space-like separated from me. If the only difference between our positions is a space-like separation, I have no reason to accept my frame of reference as constitutive of reality while excluding yours. This suggests that the primary motivation for presentism (and, I argue, for other PPR views) — the thought that there is something irreducibly special and important 
about one's own viewpoint such that it must be foundational to metaphysical reality — is arbitrary and unmotivated.

Consider the same parity objection applied to the inertial-frame option. Under this picture, when I ask a question, the relevant standpoint from which to evaluate it is the frame at which I am at rest when I ask the question and the time within that frame at which I ask it. If you are within my light-cone at the moment I ask the question, the fact that you are in my absolute past or future may give me presentist reason to exclude your standpoint. If, however, we are in relative motion and coincide at the location from which I ask the question, then the only difference between us is our relative motion (Fine 2005, p. 301). But in that case, it is unclear how the difference in our relative motion could give me reason to exclude your frame of reference from reality while admitting mine. The presentist may respond that the answer lies in an appeal to indexicality. A presentist may reject another observer's standpoint from reality because she is not here-now or is not now-at-rest (from the presentist's viewpoint). While this appeal to indexicality might be plausible for the pre-relativistic presentist, it does not have the same impact for the presentist revising her position in light of special relativity. While it may be plausible that a past observer does not occupy a standpoint of reality because she is not present, the same cannot be said for the post-relativistic presentist's appeal to indexicality. This, as Fine puts it, simply collapses to, "You do not occupy the standpoint of reality since you are not me" (2005, p. 302). The presentist's attempt to maintain their intuition in light of special relativity again leads to arbitrary and unmotivated privileging of their own standpoint, resulting in a apparent dependence of the tensed on the first-personal.

Of course, a natural objection is that, since we know that special relativity is not a final theory of physics and is thus likely to be replaced by a future theory, there is no need to ensure 
that our other theories are compatible with it. However, the case is a bit more complicated. On the one hand, followers of Kuhn (1962) hold that some scientific paradigm shifts, such as the transition from the classical to the quantum or the Newtonian to the Einsteinian, constitute such radical and revolutionary changes that the theories are incommensurable and thus there can be no theoretical content preserved in the transition. Structural realists such as French and Ladyman (2003) and Ladyman, Ross, Collier, and Spurrett (2007), however, have shown that there is often more continuity across theory change than is recognized.

Consider the transition from Newtonian gravitation to General Relativity. This shift exemplifies a radical change in ontology across the two theories but one that preserves a great deal of structural continuity. Under Newton's law of universal gravitation, gravity was thought to be an attractive force between massive objects that acted on bodies at a distance. Einstein's General Relativity dispensed with this ontological commitment, understanding gravitation to be an attribute of curved spacetime rather than a force propagated between bodies. Despite the difference in the theories' underlying ontologies, there is structural continuity between them, as Newtonian Gravity obtains in the limit of weak gravitational fields in General Relativity (Silberstein, Stuckey, \& McDevitt 2018, p. 20).

There are myriad examples of structural continuity across theory change. Quantum mechanical models mathematically reduce to classical models in the limit of large numbers of particles or the limit of Planck's constant becoming arbitrarily small (Ladyman, Ross, Collier, and Spurrett 2007). The Correspondence Principle shows that the behavior of systems described by QM reproduces classical physics in the limit of large quantum numbers. Structural continuities between classical mechanics and special relativity also exist. While the coordinate systems that arise from inertial frames of reference of stationary bodies are related by Lorentz 
transformations in special relativity rather than by Galilean transformations as they are in classical mechanics, as the value of $c$ - the velocity of light in a vacuum-goes to infinity, the mathematical structure of Lorentz transformations increasingly approximates that of Galilean transformations (Ladyman, Ross, Collier, and Spurrett 2007).

The transition from Fresnel's theory of optics to Maxwell's theory also shows retention of structure across theory change (Worrall 1989; Ladyman 1998). While the two theories differ in the interpretations and ontologies ascribed to their structural contents (e.g. light travels through an elastic solid ether for Fresnel but through an electromagnetic field for Maxwell), the differential equations remain constant during the transition from the former to the latter.

While structural realists present this argument partly in response to Laudan's (1981) pessimistic meta-induction, one need not accept structural realism in order to take the basic point of the argument: when a theory successfully makes a novel prediction, as Newtonian gravitation and Fresnel's optics did, we should expect some aspect of the theory's structural content to be retained in whatever theory replaces it. Applying this insight to the case of special relativity yields the expectation that some of its structural content, namely those features of the theory that are responsible for its successful novel predictions, will be retained in whatever theory follows it. This would include the assumption that $c$ has a non-zero value and thus that there is an upperlimit on the speed of information transfer within the universe. ${ }^{2}$

\footnotetext{
${ }^{2}$ Many physicists and philosophers take this claim to have already been straightforwardly falsified by Bell's Theorem. But again, the case is not so simple. What Bell's inequalities showed was that no local hidden variable theory can accurately reproduce the observed probabilities of his experiment. What the experiments straightforwardly show is that a number of plausible assumptions such as locality,
} 
Special relativity has extraordinary predictive power and empirical confirmation. It also works best against a backdrop of four-dimensional space-time. While space and time are sometimes pre-theoretically taken to be separate, special relativity indicates that they are different dimensions of single manifold. The presentist's effort to maintain the intuition that there is a metaphysically significant distinction between space and time illustrates the strained results of prioritizing the metaphysical fundamentality of bird-view facts over frog-view facts. Because our movement through time is more constrained than our movement through space, the presentist infers that there must be some fundamental metaphysical asymmetry between space and time. They assume that the way the world appears to us at the very limited spatio-temporal scales to which we have direct experiential access must be the way the world is metaphysically. To reject PPR about tensed facts, on the other hand, is to recognize that phenomenology of time should not be considered more metaphysically fundamental than the picture of reality indicated by the structural content of our most predictive scientific theories.

One approach to scientific metaphysics that takes the bird view as prior is Silberstein, Stuckey, and McDevitt's (2018) Relational Blockworld. Like Wharton, Miller, and Price (2011), these authors are motivated by seemingly insurmountable challenges and incompatibilities in separability, and independence are incompatible. At least one must be relinquished, but it is not clear which one. It is not straightforward that locality is violated by Bell's inequalities, as one possibility is interpreting quantum entanglement to be something that occurs outside of spacetime in Hilbert space. This is one way that the Everettian can go. Thus, one of the arguments in favor of the Everettian interpretation is that it is able to retain locality (by posting entanglement as a process that occurs in Hilbert space rather than within spacetime) and thus compatibility with special relativity's most empirically predictive foundational assumption. 
modern physics to jettison the dynamical worldview in favor of a Langrangian-first schema. They argue that the Newtonian approach to physics that takes motion and change in time to be the primary objects of physical explanation has largely failed. In its place they propose a metaphysics that accepts the Lagrangian schema, whereby the explanatory value of the leastaction principle combines with both the initial conditions of a system as well as its future states to explain the system's present state. While the Langrangian-first approach is very much in line with taking the primacy of the bird view over the frog view, Silberstein, Stuckey, and McDevitt don't quite go far enough with their own approach, as they rule out a multiverse view on the seemingly shaky grounds that it is unfalsifiable. ${ }^{3}$

In the next section, I argue that interpretations of quantum mechanics that rule out the quantum multiverse can be understood as endorsing a form of PPR, namely realism about what I call 'world-indexed' facts. I consider parallels between realism about irreducibly world-indexed facts and realism about irreducibly tensed facts. I argue that the realism about irreducibly worldindexed facts is motivated by the same overvaluing of phenomenology and intuition that is responsible for attempts to preserve presentism in light of special relativity. Thus, non-multiverse interpretations of quantum mechanics fall prey to the same methodological objections that undermine the non-naturalistic motivations of PPR more generally. Using the Copenhagen interpretation as a case study, I consider some of the ways this form of PPR is vulnerable to the

\footnotetext{
${ }^{3}$ As Carroll (forthcoming) argues, the cosmological multiverse is confirmed the same way conventional science is, via abduction, Bayesian inference, and successful empirical prediction.
} 
same objections as presentism. ${ }^{4}$ Combining a rejection of PPR about tensed facts with a rejection of PPR about world-indexed facts results in a picture that has been called the quantum block universe (Saunders 1993) and described as "a multiverse of discrete, parallel, block universes which are identical to each other up to certain points in the MWI 'trunk' before they diverge according to the MWI branching" (A. Mckenzie 2016). In what follows, I discuss paths that lead there.

\subsection{The Metaphysics of Quantum Mechanics}

Interpretations of quantum mechanics differ in how they respond to the measurement problem. The Schrodinger equation describes the evolution of quantum systems, and its dynamics are unitary and deterministic. Before measurement, a quantum system can be in a state of a superposition. If a quantum system is in a state of superposition at one time, the dynamics predict that it will be in a state of superposition at a later time. But we never seem to measure or observe quantum systems in superposed states, nor do we have much idea of what such a state would look like. When a measurement is taken, the wave function appears to instantaneously collapse, leaving a definite outcome where before there was only probability. Different interpretations of

\footnotetext{
${ }^{4}$ Copenhagen is not the only interpretation that rules out a quantum multiverse, though it is the one I focus on here as a primary example of a collapse theory that produces a PPR metaphysics. While other collapse theories avoid some of the worst problems with Copenhagen, such as those surrounding the question of when physical measurement occurs, they are still susceptible to the objections I consider here against PPR views. For instance, GRW theory is vulnerable to the same objections regarding adding ad hoc postulates to the theory in order to avoid accepting a quantum multiverse.
} 
quantum mechanics have different views on the reality of the wave function as well as different explanations of its apparent collapse.

One way to think about the measurement problem is in terms of three compelling yet conflicting principles. Ladyman and Ross (2007, p. 180) offer the following characterization of the measurement problem in terms of three intuitive principles that cannot all be true:

(1) All measurements have unique outcomes.

(2) The quantum mechanical description of reality is complete.

(3) All time evolution of quantum systems is in accordance with the Schrodinger equation.

Since these principles cannot all be true for a quantum system that is initially in a superposition of the property that is being measured, which one should be relinquished? How an interpretation of quantum mechanics solves the measurement problem plays a central role in determining its metaphysics. Since a comprehensive survey of QM interpretations is beyond the scope of this chapter, I look only at the Everett and the Copenhagen interpretations to illustrate the metaphysical issues at stake. The Everett interpretation rejects (1), which results in a multiverse or 'many worlds.' The Copenhagen interpretation and other collapse theories reject (2), which entails that quantum mechanics does not give a complete account of the dynamical properties of quantum systems.

\subsubsection{The Copenhagen Interpretation and PPR}

When Bohr (1935) first introduced the Copenhagen interpretation, he did so by way of the principle of complementarity. The principle states that an experiment may show matter to behave as a particle or as a wave, but never both at once. The Copenhagen interpretation explains the determinate nature of a property after observation via the presumed mechanism of wave-function 
collapse. Because the Copenhagen interpretation gives up (2), an additional postulate must be added to the theory to specify the conditions under which collapse occurs. The theory that collapse occurs in response to a measurement of a system being taken faces a number of wellknown problems, such as the fact that measurement is not a well-defined notion and cannot be precisely identified and applied at the quantum scale. ${ }^{5}$

Another cost of the view is that it violates the principle of locality. The principle of locality states that an object can only causally influence and be influenced by objects in its immediate surroundings, not by distant objects. Thus, locality prohibits action at a distance. Compatibility with locality (conceived as compatibility with Lorentz-invariant space-time) is what allows quantum mechanics to be unified with special relativity. Since violations of locality involve something (namely information) traveling faster than the speed of light, as would be the case if a change in some entity were to directly influence a distant object instantaneously, they render a theory incompatibility with special relativity's upper-limit on the speed of information transfer. Thus, a major cost of the Copenhagen interpretation is that its acceptance of non-locality precludes its unification with special relativity.

The Copenhagen interpretation can be understood as promoting a kind of privilegedperspective realism about world-indexed facts. A world-indexed fact is the quantum-world analog of a tensed fact. It is a fact that obtains only with respect to some world or branch within the quantum multiverse. ${ }^{6}$ While the existence of world-indexed facts follows from the Everett

\footnotetext{
${ }^{5} \mathrm{I}$ will not address this significant worry, however, as the primary concern here is with issues that this interpretation shares with other collapse theories.

${ }^{6}$ Issues related to the terminology of 'worlds' and 'branching' are addressed in the following section.
} 
interpretation, those who do not subscribe to Everett can think of world-indexed facts using quantum worlds as theoretical constructs much in the same way that Lewis's (1986a) opponents treat the objects of possible-worlds semantics.

Consider: A physicist in our world learns the potential locations of an electron before measurement by identifying the electron's wave function. Since the electron's wave function can be obtained by anyone using the same equations, regardless of which branch of quantum reality they inhabit, this reflects that the wave function represents a bird-view fact that is not dependent on one's location in a given quantum world. The physicist then takes a measurement and determines the electron's location to be at space-time point $m_{1}$. However, in other possible measurements, the electron is determined to be at locations other than $m_{1}$. When the physicist utters, "The electron is located at $m_{l}$," they are uttering a proposition whose truth is evaluated with respect to the branch or world at which it is uttered. ${ }^{7}$ This proposition therefore expresses a world-indexed fact. World-indexed facts are contrasted with those that are not indexed to any particular world. Since the proposition, "In quantum-world $q_{5}$, electron $z$ is at $m_{l}$ " obtains whether or not it is uttered in $q_{5}$, it does not express a world-indexed fact. Call the nonperspectival fact it does express a 'world-less' fact.

The four principles that Fine identifies can be used to categorize views in many areas of metaphysics. Generally, PPR views retain Realism, Absolutism, and Coherence while relinquishing Neutrality; and anti-realist views about perspectival facts give up Realism while retaining Neutrality, Absolutism, and Coherence. This outline of metaphysical commitments can help to illuminate some of the differences between various interpretations of quantum

\footnotetext{
${ }^{7}$ See Wilson (2011) for an account of the formal semantics that this picture involves.
} 
mechanics, specifically between collapse theories and no-collapse theories. In the next section I suggest that implicit in collapse theories (and specifically in non-multiverse interpretations of quantum mechanics) is a form of PPR—specifically, about world-indexed facts.

If one were to extend Fine's four principles to possible views about world-indexed facts, they might look like this:

1. Realism - Reality is constituted at least in part by irreducibly world-indexed facts.

2. Neutrality—No world is metaphysically privileged over any other.

3. Absolutism-The composition of reality is an absolute matter, i.e. not relative to a world.

4. Coherence-Reality is not contradictory, it is not constituted by facts with incompatible content.

In terms of Fine's principles, the Everett interpretation denies Realism about world-indexed facts and retains Absolutism, Coherence, and Neutrality. In this way, it is analogous to eternalism, which denies Realism about world-indexed facts while retaining Absolutism, Coherence, and Neutrality. The Copenhagen interpretation, on the other hand, retains Realism about world-indexed facts along with Absolutism and Coherence and denies Neutrality. In this way it is similar to presentism, which also maintains Realism, Absolutism, and Coherence but denies Neutrality.

The theoretical parallels among PPR about world-indexed facts and other privilegedperspective realisms are striking. PPR about world-indexed facts accepts Realism (about perspectival facts), Absolutism, and Coherence, while rejecting Neutrality. It accepts that there is a metaphysically privileged standpoint of reality, and that standpoint is this quantum world. The fact that PPR about world-indexed facts rejects Neutrality reflects its acceptance of frog-view 
facts as fundamental constituents of reality. By assuming that consistency with the frog perspective takes precedence over theoretical considerations, the Copenhagen interpretation limits the scope of metaphysical reality to include only what is observable in this quantum world. Just as the ontic presentist limits their ontology to what exists from their own temporal perspective, the Copenhagen theorist limits their metaphysical reality to what obtains from their location in the set of quantum worlds. The view that metaphysical reality contains irreducibly world-indexed facts that cannot be expressed by any world-less facts is PPR about worldindexed facts. This view forms part of the metaphysical picture of the Copenhagen interpretation.

The Copenhagen interpretation posits a set of postulates indicating that the true description of metaphysical reality obtains from a privileged perspective. The Copenhagen interpretation's claim that only the experimentally observable constitutes reality is equivalent to the claim that only what is observable from within this quantum world constitutes reality. The Copenhagen interpretation takes the reality that is apparent to us as the inhabitants of a single quantum world to be the whole of metaphysical reality. This view denies that physical reality could extend beyond what we experience and have practical access to. ${ }^{8}$ Contrast this with the Everettian ascription of fundamentality to the bird view, which takes the wave function to be a fundamental component of metaphysical reality. What the Everettian takes to follow from this-that all outcomes denoted by the wave function are equally real—is just what the Copenhagen theorist rejects in order to maintain the privileged perspective of this world over those of other quantum worlds.

${ }^{8}$ Tegmark (2010, p. 578) refers to the rejection of this possibility as the "Omnivision assumption: physical reality must be such that at least one observer can in principle observe all of it." 
The Copenhagen interpretation faces some of the same challenges that threaten factive presentism. Just as special relativity implies that there can be no physical reason to privilege any spatio-temporal perspective as more metaphysically real than any other, nothing in the quantum wave function or in quantum theory itself indicates that there is anything more metaphysically real about the outcome we observe than the other outcomes reflected by the probability amplitude of the wave function. Both presentism and the Copenhagen interpretation posit the existence of a metaphysically privileged perspective that does not come directly out of the theory but must be added in by hand in order to 'save' our experience.

A tenet of scientific realism is that we have reason to believe in the physical reality of mathematical structures posited by scientific theories when they successfully produce novel predictions and explanations. Berenstain (2016) illustrates how, for instance, the mathematical formalism of quantum incompatibility predicts that whenever a physical system is in an eigenstate of one of a pair of quantum properties it will always be in a superposition of the other. ${ }^{9}$ The higher-order property of incompatibility of two such properties is defined in terms of the non-commutativity of their corresponding operators. The mathematical structure that describes the relations of these properties is responsible for the measurable prediction that when two observables are incompatible they will not both be simultaneously instantiated with determinate values by a single system. The quantum wave function and Schrodinger dynamics are also mathematical structures that play a central role in producing the enormous empirical

\footnotetext{
${ }^{9}$ The linear algebra predicts that it will not be possible to simultaneously measure definite values for two incompatible observables in a quantum system. For instance, since the operators corresponding to spin in the x-direction and spin in the y-direction do not commute, they represent incompatible observables.
} 
success of quantum theory. As Deutsch emphasizes, "The formalism, of quantum theory did not come out of nowhere. It is the solution of a scientific problem and, as always in science, the problem was not primarily what mathematical formula best predicted the outcomes of experiments. It was what mathematical structures correspond best to reality" $(1996,227)$. The Copenhagen interpretation does not ascribe physical reality to these mathematical structures that have effectively produced both prediction and explanation of observable phenomena. Instead it treats facts determined through observation to be the fundamental constituents of reality, seemingly renouncing scientific realism about some of quantum theories most empirically successful entities.

Like other interpretations of scientific theories driven by a need to maintain a version of PPR, the Copenhagen interpretation elevates the way the world appears to be to the internally located observers above the way that our best theories suggest it is. The interpretation treats the scope of information obtained through observation as metaphysically universal while limiting the scope of information derived from the mathematical structure of the wave function. The view privileges facts that obtain from the frog perspective as more fundamental than those that obtain from the bird perspective. Like presentism, then, the Copenhagen interpretation seems to undervalue the role that the mathematical structure of an empirically successful theory can play in accessing the modal, physical, and metaphysical nature of the universe. ${ }^{10}$

\subsubsection{The Everett Interpretation: Neutrality and the Multiverse}

\footnotetext{
${ }^{10}$ See Berenstain (2017) for a discussion of the relationship between mathematical structure and the modal structure of the physical world.
} 
Contrast the Copenhagen interpretation and its resulting PPR metaphysics with the metaphysics of the Everett interpretation. Named after Hugh Everett, who first introduced what was known as the "Relative-State formulation" of quantum mechanics (Everett 1957), the view was later defended and renamed the "Many-Worlds" interpretation by DeWitt and Graham (1973). Carroll and Singh (2018) take the Everettian interpretation to offer the "most pure, minimal" quantum ontology, which includes only the stripped-down elements of a vector in Hilbert space and a Hamiltonian, from which everything else emerges. Deutsch $(1996,2010)$ objects to the fact that it is even called an interpretation rather than simply acknowledged as the theory of quantum mechanics itself - no interpretation necessary. While other interpretations require the addition of ad hoc postulates to quantum theory in order to redeem intuitions about the uniqueness of the universe, the Everett Interpretation, as Wallace $(2013,212)$ describes it, "is a pure interpretation of quantum mechanics. It leaves the quantum formalism, dynamics and state space alike, completely alone." In other words, it is the view that "quantum mechanics is everything, and it is right" (Wallace 2013, 212). Nothing more needs to be added to the quantum formalism, which has already produced an enormously empirically successful theory, to get a complete story of quantum phenomena.

A central feature of the Everett interpretation is that QM is a unitary theory, as all isolated systems evolve according to the Schrodinger equation. The Everett interpretation thus maintains the determinism of quantum mechanics. When a system in a state of superposition is measured, there is no assumption of a unique definite outcome and there is no presumption of wavefunction collapse (Tegmark 2010). The wave function is considered to be a genuine physical entity. The result of a measured observation is just one part of the unitary quantum state represented by the wave function. Though all of the probabilistic results occur in different 
branches of the quantum multiverse, only one outcome is manifest in each branch and thus only one is observable in each world. The wave function appears to collapse to the observer by way of quantum decoherence while in fact retaining its physical reality. While the observer sees only one experimental result, all of the possible outcomes denoted by the wave function occur in different worlds that are likely practically inaccessible to the observer in this world because of the rate at which they are expanding away from one another. ${ }^{11}$

While different possible outcomes for a physical event or experiment occur in other branches of the physical universe, as Tegmark (2010) argues, the assumption that the view entails some sort of "splitting" or "branching" of the universe as an event occurring within time is a misrepresentation of the theory. This sentiment echoes Saunders's claim in his early description of what he refers to as the "quantum block universe." Saunders writes, "The notion of 'splitting' is particularly inapposite... (no such process is defined in the block universe); nor is our world subject to "splitting," no more than "now" contains different times." Saunders 1993, p. 1563). While "branching" language is commonly used in discussions of the Everett interpretation, nothing in the theory mandates an assumption that this occurs as an event within a background of time rather than the assumption that the multiverse has a branched structure that is already determined within something like a background eternalist framework.

The version of the Everett interpretation that is most compatible with the bird view is one that takes the multiverse to be made up of various world-states combining to produce world-lines in a structured graph network that instantiates a branched structure, producing what can then be

\footnotetext{
${ }^{11}$ For arguments that the quantum multiverse and the cosmological multiverse may well be one and the same, see Boddy, Carroll, and Pollack (2017) and Tegmark (2008).
} 
referred to as the quantum block multiverse. Fundamentally then, on this picture, there is just one thing in our quantum ontology—-the universal quantum state. In a block multiverse picture, nothing changes and nothing branches; worlds are simply ordered. Some world-states are accessible from other world-states while others are not.

I follow Wilson's (2011) suggestion that the Everettian employ the language of the multiverse in order to pick out relevant parts of the universal quantum structure in metaphysical theorizing. He writes,

At the fundamental level, the ontology is monistic - there is just one single highlystructured object, the universal quantum state. Call the language which describes reality in these terms the Universal State language. By use of decoherence theory to pick out privileged structure from the universal state, we can construct a language (call it the Pluriverse language) in which we quantify over structural features of the universal state: branches, branch segments, and so on. The Pluriverse language can be thought of as the working language of metaphysical theorizing for Everettians. (2011, p. 18)

The language of 'worlds' and 'branches' is used interchangeably to identify locations within the multiverse, and the multiverse can be taken to refer to the universal quantum state. Quantum worlds sometimes have overlapping parts with other quantum worlds, just as branches on a tree sometimes share parts with other branches on the same tree. Smaller branch chunks may be referred to as 'branch segments,' etc.

This quantum block version of the Everett interpretation avoids the metaphysical problems associated with interpretations of quantum mechanics theoretically motivated by frogperspective considerations like many collapse interpretations. The view maintains the metaphysical priority of the bird-view over the frog-view, thus avoiding the objections that PPR interpretations face. I now turn to two common objections to the Everett interpretation and 
suggest that reconsidering them within a framework that privileges the bird perspective can resolve many of the apparent issues.

\subsubsection{The Objection from Ockham's Razor}

A tempting objection to multiverse metaphysics is that it posits an extraordinarily high number of unnecessary worlds in order to make sense of quantum theory. This is a clear violation of Ockham's razor, as the objection goes. However, it is not clear at all that Ockham's razor tells against rather than in favor of a multiverse picture. On some readings of Ockham's razor, the Everett interpretation comes out ahead. As Baker (2003) notes, a distinction is often made between syntactic simplicity, which captures the number and complexity of hypotheses or axioms in a theory, and ontological simplicity, which considers the number and complexity of things posited. Baker refers to minimizing the former as elegance and to minimizing the latter as parsimony. On the measure of syntactic simplicity, the Everett interpretation is hard to beat. It takes the formalism of QM at face value and avoids adding ad hoc fundamental postulates, as so many collapse theories must. GRW, for instance, must introduce a new fundamental constant, which is a serious cost to the interpretation's syntactic simplicity.

Those who object to the quantum multiverse on the basis of Ockham's razor prioritize ontological simplicity over syntactic simplicity, parsimony over elegance. This choice of theoretical values can be understood as stemming from a privileging of the frog perspective over the bird perspective. An example from computation reveals that it is often far simpler, i.e. it takes fewer bits of information, to produce all the entities in an infinitely large set than to produce only a single member of a set. In computation, the algorithmic content of a number is defined as the length in bits of the shortest computer program that can produce that number as output. 
However, the set of all integers can be generated by a trivial computer program (Tegmark 2008, p. 12). The algorithmic content of the entire set is much smaller than that of even a single element of the set. The standard of ontological parsimony applies to that which results from a theory, while syntactic simplicity applies to the algorithm required to produce that ontology. In this case, the theory that posits the existence of an infinitely large set of objects is less computationally complex than one that posits the existence of only a single member. These two theoretical virtues come apart and often pull in opposite directions. Applying simplicity to the output of an algorithm — which is what ontological parsimony measures-reflects the assumption that the frog perspective has metaphysical primacy. Whereas applying simplicity to the axioms required to produce the output—what syntactic simplicity measures—reflects a view on which the bird perspective is taken to be metaphysically fundamental.

So parsimony and elegance are often in conflict, and the Everett interpretation has an advantage over non-multiverse interpretations of QM on syntactic simplicity. But is a quantum multiverse picture actually worse off on measures of parsimony than a non-multiverse picture? One common line of reasoning is that ontological parsimony only applies to types of entities rather than to tokens. While some, such as Nolan (1997b) and Cowling (2013), argue for a further distinction between qualitative and quantitative parsimony, the latter's status as a theoretical virtue is much more contentious than the former. Lewis (1973, p. 87), for instance accepted the theoretical virtue of qualitative parsimony but recognized no such presumption in favor of quantitative parsimony. On a view like his, a theory that posits the existence of numbers as a new type of entity is ceteris paribus less ontologically parsimonious than one that does not. But a theory that posits only even numbers is not more ontologically parsimonious than one that posits all the natural numbers. By this measure of parsimony, the Everett interpretation is no 
worse-off than non-multiverse pictures, since it isn't positing any new type of entity. We already have a world, and the theory just posits more of them. Since it does not commit us to any new sort of thing or unique substance, it is not worse off than non-multiverse theories with respect to qualitative parsimony.

If quantitative parsimony is a consideration, it is presumably one that tells against a multiverse picture. On the other hand, qualitative parsimony may actually tell in favor of an Everettian multiverse. The multiverse that follows from the Everett interpretation would be more qualitatively parsimonious than the single-universe picture, for instance, it allows us to do away with the qualitative distinction between the physically possible and the physically actual or even between the physical and the mathematical as Tegmark (2008) has argued. It is thus by no means obvious that considerations related to Ockham's Razor constitute an objection to a multiverse metaphysics motivated by the Everett interpretation of QM.

\subsubsection{Objection from Indistinguishable Subjective Copies}

Another family of objections to the Everettian multiverse turns on a series of seeming misunderstandings about the metaphysical implications of subjectivity, first-personal facts, and self-locating uncertainty. Consider, for instance, Albert and Loewer's (1988) explanation of their objection to the Everett interpretation based on issues of subjective conscious minds, introspection, mental states, and personal identity.

The heart of the problem is that the way we conceive of mental states, beliefs, memories, etc., it simply makes no sense to speak of such states or of a mind as being in a superposition. When we introspect following an $\mathrm{x}$-spin measurement we never, as apparently predicted by the theory, find ourselves in a superposition of thinking that spin is up and thinking that spin is down. If introspection is to be 
trusted, and it seems part of our very concept of mental states that it is trustworthy at least to this extent, then we are never in such superpositions (p. 203).

Albert and Loewer construct an argument that any many-worlds interpretation has to be committed to non-physicalism because mental states won't supervene on brain states. Their argument turns on the assumption that for any observer in a multitude of brain states across the multiverse, there will only be one mental state that they can be in. They take this view to be "especially pernicious" since "it entails that mental states do not even supervene on brain states (or physical states generally) since one cannot tell from the state of a brain what its single mind believes" (206). This understanding assumes that there is a further question that the Everett interpretation gives rise to but doesn't have the tools to answer: which of these physical observers is the subjective mind?

In many ways, these issues seem to boil down to the same questions and confusions that arise for Parfit (1971) about personal identity in any context, not just within a branching quantum multiverse. The problem of fission for personal identity captures the same concern that Albert points to in the Everettian context: which one of these people/minds/counterparts is really me? Indeed, Papineau (1996) suggests that the Everettian can straightforwardly invoke Parfit's account of personal survival as opposed to personal identity. His work demonstrates "how to talk coherently" about a conception of self in terms of branching experiential world-lines made of overlapping pasts that eventually diverge in physically indistinguishable copies. Given that Parfit has demonstrated the coherence of this hypothesis, Papineau writes, “I don't see what else argues against it, except its unfamiliarity" (p. 237).

Carroll and Sebens (2014) warn against what they take to be the naïve reaction that selflocating uncertainty poses a crisis for multiverse accounts. They take self-locating uncertainty to 
be a generic and expected feature of human cognition in a multiverse in which there is a temporal lag, however brief, between branching and post-measurement updated knowledge states. They argue that the Born rule can be derived from quantum mechanics given assumptions about the uniquely rational way for an observer to apportion credences in the time between wave function branching and the observer's knowledge updating based on registering the measurement outcome. They explain, "In our approach, the question is not about which observer you will end up as; it is how the various future selves into which you will evolve should apportion their credences."

Albert and Loewer's worry about personal identity seems to arise from an assumption of PPR about world-indexed facts. Specifically, the view commits to PPR about world-indexed facts that locate a specific observer-copy and index their specific conscious experience to a single universe or location within the quantum multiverse. This move works as an explication of their view but not as an objection to the Everett interpretation, as it simply begs the question against the anti-realist about irreducibly world-indexed facts. The anti-realist in both cases suggests that such a question can only be asked from within a certain standpoint, either from within a single branch or universe within the quantum multiverse, as is the case for worldindexed facts, or from within a specific frame of reference plus a time, as is the case for tensed facts. Beyond that, for the anti-realist, there is no further substantive question to be asked. ${ }^{12}$

\footnotetext{
12 That Albert and Loewer take there to be a further question of which person or physically indistinguishable quantum counterpart is really them further draws out the analogy between PPR about world-indexed facts and PPR about tensed facts. The presentist looks at the description of the world that arises from a complete set of tenseless facts and takes there to be a further unanswered metaphysical question: which one of these times is really now?
} 
Deutsch (1996, p. 223) explains the ambiguities of ordinary language that give rise to the confusion that Albert and Loewer demonstrate by suggesting that two apparently conflicting reports of what an observer is seeing - one report of seeing tea, the other of coffee, both of which are true - are to be understood as merely a special case of a general multiplicity in physical reality writ large. He writes,

I have just said that I cannot see the coffee, and that I am having the perception of seeing the coffee. This is no contradiction, merely two different uses of the word ' $I$ '. The problem here is that ordinary language implicitly makes the false assumption that our experiences (and observable events in general) have a singlevalued history. To help resolve the ambiguities created by this assumption, Lockwood introduces the term Mind to denote the multiple entity that is having all the ('maximal') experiences that I am in reality having, and reserves the term mind for an entity that is having any one of those experiences. So I (the Mind) am both seeing tea and seeing coffee, and am simultaneously reporting both experiences, but I (the mind), who am writing 'tea' am seeing only tea. Similarly, we call multivalued physical reality as a whole the multiverse, to distinguish it from the universe of classical physics in which observables can take only one value at a time. (p. 226)

Deutsch embraces Lockwood's (1996) use of capitalization to distinguish between the two under-specified uses of 'I' and 'mind.' Deutsch and Lockwood's use of 'Mind' is evaluated from the bird perspective, as it quantifies over the domain of the entire multiverse, while 'mind' is evaluated at and indexed to a single quantum world.

Given this clarification, it seems that the only way to maintain Albert and Loewer's objection is by claiming that there can simply be no physically indistinguishable copies of subjective observers. Tegmark (2010) discusses a dubious assumption on which objections to the quantum multiverse are often based which further brings out the intuition that motivates Albert and Loewer's objection. Tegmark identifies what he refers to as the "no-copy assumption," that "no physical process can copy observers or create subjectively indistinguishable observers" 
(578). This possibility is unacceptable for Albert and Loewer because they have already concluded that it would leave a well-defined question unanswerable. Albert and Loewer's confidence that the question of which physically and subjectively indistinguishable quantum counterpart is really the observer can be diagnosed as the same sort of certainty in our special and unique nature that has long motivated resistance to paradigm-shifting changes in scientific worldviews.

\subsection{Privileged Perspectives and Induction over the History of}

\section{Science}

The history of modern science can be viewed as a series of discoveries that have continually dethroned humankind from a presumed special and unique place in the universe. The transition away from Ptolemy's geocentric model and the corresponding Aristotelian worldview which posited that the sun and the planets revolved around Earth in circular orbits exemplifies this. Ancient Greek and Roman astronomers believed the circle to be the most perfect shape and thus assumed that celestial bodies must move in a circular motion as the heavens were perfect and unchanging. When observations such as Mercury's retrograde motion created challenges for this theory, astronomers postulated the existence of a complex and arbitrary system of epicycles and deferents to account for differences in orbit in order to retain the Ptolemaic picture. Astronomers posited ad hoc mechanisms to save what they thought must be the correct theory and resist the transition to the Copernican heliocentric theory. The Copernican model removed Earth and thus humanity from its presumptive place at the center of the cosmos and cast it into the realm of ordinary planetary bodies orbiting the sun. 
Cosmology is not the only area of science in which our intuitions fall short of grasping the nature of reality, however. Biology is another. The transition from The Great Chain of Being picture to Darwin's theory of evolution by natural selection showed that humans could no longer take themselves to have been divinely created apart from the biological processes that produced other lifeforms. Darwin's theory unified the processes that produced the origins of humanity with those that created other animal species. Humanity was again removed from its presumed pedestal in nature.

In modern physics, Einstein overturned the accepted cosmological assumption that Newtonian mechanics was the ultimate physical theory that applied to the entirety of the universe. Whereas human experience of space, time, and length gave rise to the presumption that these quantities were invariant and absolute, Einstein's theory of special relativity showed them to be dependent on the observer's frame of reference. While the spatio-temporal interval remained invariant in his theory, the other quantities became relative to an inertial frame. The reach of phenomenological intuition was once again shown to exceed its grasp. The presumed metaphysical uniqueness of the continuously moving now could no longer be retained. The moment in time that we inhabit was shown to be nothing more than one moment among many, all equally real within the space-time manifold that structures them. PPR about tensed facts became untenable.

Now once again, as the Everett interpretation suggests that we live in a quantum multiverse filled with indistinguishable copies of ourselves, many are affronted by the sense that quantum multiverse has removed the specialness and perceived ineffability of our individual subjectivity. These are indeed psychologically challenging scientific discoveries, but the fact that they are difficult for us to reconcile is not an argument against them. Deutsch (1996, p. 226) suggests this 
psychological difficulty underlies many instrumentalist interpretations of wildly successful scientific theories, from the Catholic Church's permission to use heliocentric theory "purely as a means of predicting astronomical observations, but not if it was interpreted as a factual theory of where and what the planets and the Earth are" to interpretations of quantum mechanics that stop short of acknowledging the quantum multiverse. What "these miscellaneous revisionist views of scientific theories have in common," Deutsch charges, "is a loss of philosophical nerve." Each of these times that humans have prioritized the frog view over the bird view, we have held on to failing worldviews at the expense of new developments in science.

We have significant inductive evidence that PPR fails. Each of these scientific paradigm shifts has demonstrated that taking the frog perspective to have metaphysical priority is an obstacle to achieving greater scientific understanding of the world we live in. Science has progressively shown that our position in the world is not special. We do not live at the center of the universe. We do not live under special terrestrial laws that differ from the laws that govern the celestial sphere. We did not come into being by processes that differ from those that produced the rest of the animal kingdom. Relativity shows that no time is special and that all times exist. 'Now' is merely indexical like 'here.' Given the success of quantum mechanics and the fact that the Everett interpretation is the theory in its purest form, perhaps we should accept that the apparent difference between the physically actual and the physically possible is merely indexical as well. ${ }^{13}$

13 Lewis (1986b) argues for this claim in his On the Plurality of Worlds, but he gets there via a route of apparent IBE from semantic theory rather than from quantum theory. His final picture is also radically different from the quantum multiverse, as it is characterized by concrete possible worlds generated 


\subsection{Evolution and Intuition, Scope and Scale}

The motivation for many PPR views, including PPR about tensed and world-indexed facts, is the intuition that the way the world appears to be from within the frog perspective reflects fundamental metaphysical reality. The advocate of PPR in both the tensed and world-indexed cases insists not just that metaphysical reality makes sense of our experiences of mid-level macroscopic phenomena, but that reality must be the way it is portrayed by our mid-level macro experiences at all other scales as well. Rather than taking the content of our experiences to apply only to the limited domains and scales that we are actually capable of experiencing, the PPR advocate takes the content of our experiences to be unrestricted in scope and universal in scale. The mistake of the privileged-perspective realist is to weight the picture of reality painted by our intuitions so heavily that empirical and theoretical investigations must be brought in line with that picture rather than vice versa. Since our direct experiences say nothing about the way the universe is at quantum or cosmological scales, it is a mistake to project the content of our midlevel macro experiences onto the world at those scales. Similarly, our experiences of mid-level macroscopic phenomena are silent on whether a quantum multiverse exists; nothing in our experience is incompatible with the existence of a multiverse. To deny the quantum multiverse on the basis of experience is also to project the content of experience beyond its domain and presume that it is universal in scope.

There is a naturalistic explanation for the inductive failure of our intuitions when it comes to discovering the metaphysical nature of reality. Ladyman and Ross (2007) have noted that our

from the Principle of Recombination of properties limited only by logical possibility, which bear no spatio-temporal relations to one another. 
intuitions about the physical world are well-developed to understand the mid-level macro scale of physical phenomena that made up our ancestral environments and that continue to constitute the scale at which humans experience the universe. But we do not have any naturalistic reason to think that our pre-theoretic intuitions about the quantum and cosmological track metaphysical reality at those scales.

People are probably also relatively reliable barometers of the behavioural patterns of animals they get to spend time observing, at making navigational inferences in certain sorts of environments (but not in others), and at anticipating aspects of the trajectories of medium-sized objects moving at medium speeds. However, proficiency in inferring the large-scale and small-scale structure of our immediate environment, or any features distant from the parts of the universe distant from our ancestral stomping grounds, was of no relevance to our ancestors' reproductive fitness. Hence, there is no reason to imagine that our habitual intuitions and inferential responses are well-designed for science or for metaphysics. (Ladyman and Ross 2007, p. 2)

Metaphysics that aims to make sense of our best physical theories should not be expected to save 'common-sense' intuitions. The frog view is pragmatically and instrumentally valuable for navigating the physical world, but we should not mistake our limited inside view of the universe for its metaphysical nature. We have better-developed ways of learning about the universe at the quantum and cosmological scales than relying on intuitions that stem from our phenomenology of the comparatively tiny range of scales which human experience is capable of capturing. ${ }^{14}$

14 Ladyman \& Ross (2007: 11) emphasize how severely limited the range of scales of human experience is compared to the range of scales at which the universe is structured. They write, "We occupy a very restricted domain of space and time. We experience events that last from around a tenth of a second to years. Collective historical memory may expand that to centuries, but no longer. Similarly, spatial 
Tegmark (2010, p. 12) echoes Ladyman and Ross's point when he writes, "Evolution endowed us with intuition only for those aspects of physics that had survival value for our distant ancestors, such as the parabolic trajectories of flying rocks. Darwin's theory thus makes the testable prediction that whenever we look beyond the human scale, our evolved intuition should break down." The history of science is filled with an overwhelming number of cases that demonstrate the limits of our capacities to intuitively discover the nature of physical reality. We don't just know that our intuitions have failed, evolutionary biology offers an explanation of why we ought to expect them to fail in such cases.

Tegmark (2010) critiques what he calls the "pedagogical reality assumption," which is the assumption that "physical reality must be such that all reasonably informed human observers feel they intuitively understand it" (p. 578). Rejection of the multiverse interpretation of QM is in the lineage of other metaphysical theories that attempt to force science to fit with our pre-theoretic, 'common-sense' intuitions about the way reality should be. The metaphysical presumption of wave function collapse as physical reality is motivated by the desire to make the facts that obtain from the frog view part of the ultimate description of reality. The Everett interpretation can maintain the apparent reality of wave function collapse while reducing frog-view facts to worldless facts about the wave function. When there are clear tensions between the picture of reality

scales of a millimetre to a few thousand miles are all that have concerned us until recently. Yet science has made us aware of how limited our natural perspective is. Protons, for example, have an effective diameter of around $10^{-15} \mathrm{~m}$, while the diameter of the visible universe is more than $10^{19}$ times the radius of the Earth. The age of the universe is supposed to be of the order of 10 billion years. Even more homely sciences such as geology require us to adopt time scales that make all of human history seem like a vanishingly brief event." 
suggested by our best physical theories and the picture suggested by our intuitions, the latter ought to be the one relinquished.

\subsection{Conclusion}

I have considered a number of problems with the practice of offering metaphysical interpretations of scientific theories that are grounded in an ascription of metaphysical fundamentality to the frog perspective. I have argued, using the examples of special relativity and quantum mechanics, that there are structural similarities in PPR metaphysical interpretations of scientific theories across domains of theoretical physics. Conceived in terms of which combination of Fine's principles a metaphysical view accepts, the structural similarities among PPR views reflect an overvaluing of the first-personal and subjective and create vulnerability to objections from arbitrariness due to their rejection of Neutrality. I have suggested that much of the motivation for PPR metaphysics is simply psychological, and I have demonstrated that metaphysical assumptions that are motivated by psychological tendencies are difficult to make compatible with induction over the history of science. Such assumptions should therefore not be driving views in the metaphysics of science, especially, as I have argued, in either quantum or cosmological domains. 


\section{Works Cited}

Albert, David \& Loewer, Barry (1988). Interpreting the many-worlds interpretation. Synthese 77: 195-213.

Baker, Alan (2003). Quantitative parsimony and explanatory power. British Journal for the Philosophy of Science 54 (2):245-259.

Berenstain, Nora (2017). The Applicability of Mathematics to Physical Modality. Synthese 194 (9): 3361-3377.

Berenstain, Nora (2016). What a Structuralist Theory of Properties Could Not Be. In Anna Marmodoro \& David Yates (ed.), The Metaphysics of Relations. Oxford University Press.

Boddy, Kimberly, Sean Carroll, and Jason Pollack (2017). How Decoherence Affects the Probability of Slow-Roll Eternal Inflation, Physical Review https://arxiv.org/abs/1612.04894

Bohr, Neils. (1935) "Can a Quantum-Mechanical Description of Reality be Considered Complete?” Physical Review 48: 696-702.

Carroll S.M., Sebens C.T. (2014) Many Worlds, the Born Rule, and Self-Locating Uncertainty. In: Struppa D., Tollaksen J. (eds) Quantum Theory: A Two-Time Success Story. Springer, Milano

Carroll, Sean M. (forthcoming). Beyond Falsifiability: Normal Science in a Multiverse. In Richard Dawid, Radin Dardashti \& Karim Thebault (eds.), Epistemology of Fundamental Physics: Why Trust a Theory?Cambridge, UK: Cambridge University Press.

Carroll, Sean and Ashmeet Singh, 2018, "Mad-Dog Everettianism: Quantum Mechanics at Its Most Minimal," submission to the Foundational Questions Institute Essay Competition, "What Is Fundamental?" https://arxiv.org/pdf/1801.08132.pdf

Cowling, Sam (2013). Ideological parsimony. Synthese 190 (17):3889-3908.

Deutsch, David. (2010) “Apart from Universes.” In Simon Saunders, Jonathan Barrett, Adrian Kent \& David Wallace (eds.), Many Worlds?: Everett, Quantum Theory \& Reality. Oxford University Press.

Deutsch, David. (1996). Comment on Lockwood. The British Journal for the Philosophy of Science Vol. 47, No. 2 pp. 222-228.

DeWitt, B. and N. Graham. (1973). The Many-Worlds Interpretation of Quantum Mechanics, Princeton University Press, Princeton.

Everett III, Hugh. (1957). 'Relative State Formulation of Quantum Mechanics', Reviews of Modern Physics 29, 454-62. 
Fine, Kit. (2005). “Tense and Reality." Modality and Tense: Philosophical Papers. New York: Oxford University Press.

French and Ladyman (2003) "Remodelling Structural Realism: Quantum Physics and the Metaphysics of Structure," Synthese 136:31-56.

Harrington, James (2008). Special relativity and the future: A defense of the point present. Studies in History and Philosophy of Science Part B: Studies in History and Philosophy of Modern Physics 39 (1):82-101.

Hut, Piet, Mark Alford, and Max Tegmark. (2006). "On Math, Matter, and Mind." Foundations of Physics 36 (6): 765-794.

Kuhn, Thomas (1962). The Structure of Scientific Revolutions. Chicago: University of Chicago Press.

Ladyman, James (1998) “What is Structural Realism?” Studies in the History and Philosophy of Science 29.3: 409424

Ladyman, J., Ross, D., Spurrett, D., \& Collier, J. (2007.) Every thing must go:

Metaphysics naturalised. Oxford: Oxford University Press

Laudan, Larry. (1981) “A Confutation of Convergent Realism,” Philosophy of Science, 48.1: 1949.

Lewis, D. (1973). Counterfactuals. Oxford: Basil Blackwell.

Lewis, David K. (1986). On the Plurality of Worlds. Wiley-Blackwell.

Lockwood, M. 1996, "Many Minds Interpretations of Quantum Mechanics", British Journal for the Philosophy of Science 47(2): 159-188.

McKenzie, Alan (2016). Some Remarks on the Mathematical Structure of the Multiverse. https://arxiv.org/abs/1602.04247.

McTaggart, J.M.E. (1908). “The Unreality of Time.” Mind 17 (68):457-474.

Nolan, Daniel (1997). Quantitative parsimony. British Journal for the Philosophy of Science 48 (3):329-343.

Papineau, David. (1996). Many Minds are No Worse than One. British Journal for the Philosophy of Science 47 (2):233-241.

Parfit, Derek (1971) Personal Identity. Philosophical Review 80:3-27.

Putnam, Hilary (1967). Time and Physical Geometry. Journal of Philosophy 64 (8):240-247. 
Simon Saunders, (1993) "Decoherence, Relative Stats, and Evolutionary Adaptation" Foundations of Physics 23 (12): 1553-1585.

Saunders, Simon (2002). How Relativity Contradicts Presentism. Royal Institute of Philosophy Supplement 50:277-.

Silberstein, M., Stuckey, W. M., \& McDevitt, T. (2018). Beyond the dynamical universe:

Unifying block universe physics and time as experienced.

Stein, Howard (1991). On relativity theory and openness of the future. Philosophy of Science 58 (2):147-167.

Stein, Howard (1968). On Einstein--Minkowski space--time. Journal of Philosophy 65 (1):5-23.

Tegmark, Max (2010). Many Worlds in Context. In Simon Saunders, Jonathan

Barrett, Adrian Kent \& David Wallace (eds.), Many Worlds?: Everett, Quantum Theory \& Reality. Oxford University Press.

Tegmark, Max (2008). The Mathematical Universe. Foundations of Physics 38 (2):101-150.

Vonnegut Jr., Kurt. (1969). Slaughterhouse-Five. Delacourt Press.

Wallace, David. (2013). A Prolegomenon to the Ontology of the Everett Interpretation. In , Alyssa \& David Z Albert (eds.) The Wave Function: Essays on the Metaphysics of Quantum Mechanics.

Wallace, David (2001). Worlds in the Everett interpretation. Studies in History and Philosophy of Science Part B: Studies in History and Philosophy of Modern Physics 33 (4):637-661.

Wharton, Ken, D.J. Miller, and Huw Price (2011). Action Duality: A Constructive Principle for Quantum Foundations. Symmetry 3: 524-540.

Wilson, Alastair (2011). Macroscopic ontology in Everettian quantum mechanics. Philosophical Quarterly 61 (243):363-382.

Worrall, John, “Structural Realism: The Best of Both Worlds?” Dialectica 43.1-2: 99-124 